\title{
Magnetization curves of plastically deformed Fe metals and alloys
}

\author{
S. Takahashi, ${ }^{\text {a) }}$ J. Echigoya, and Z. Motoki \\ Faculty of Engineering, Iwate University, 020-8551 Morioka, Japan
}

(Received 19 April 1999; accepted for publication 7 October 1999)

\begin{abstract}
The hysteresis loop of plastically deformed $\mathrm{Fe}$ metals and alloys was measured for single crystals, polycrystalline iron, and A533B steel samples, and the dislocation density and structure of these samples were observed by electron microscopy. The relation between structure-sensitive properties and applied stress $\sigma$ was analyzed in connection with grain boundaries and dislocations. The coercive force $H_{c}$ increases with $\sigma$ and is represented by one curve in all the samples. The magnetic susceptibility $\chi_{c}$ above the coercive field can be described by a simple relation to the magnetic field $H$. $\chi_{c}=c / H^{3}$ in a limited region of $H$. The parameter $c$ depends only on lattice defects such as dislocations and the grain size and has a simple relation to them, but is independent of the kinds of samples or of the process of plastic deformation. The susceptibility $\chi_{c}$ is explained in comparison with the susceptibility $\chi_{r}$ in the range of approach to saturation. The magnetic properties $c$ and $H_{c}$ are discussed considering the dislocation density and its distribution including the effect of grain boundaries. These magnetic properties are useful for nondestructive tests of metal fatigue. (C) 2000 American Institute of Physics. [S0021-8979(00)01102-6]
\end{abstract}

\section{INTRODUCTION}

The currently employed nondestructive tests for metal fatigue are useful in the investigation of cracks and fractures in the materials, such cracks being produced by the pile of dislocations, where the dislocation density $\rho$ is maximum locally. The work-hardening process has long been studied from the viewpoint of dislocation theory and we can obtain detailed information on dislocations as related to metal fatigue. However, few investigators have conducted nondestructive tests before the appearance of cracks. Nondestructive tests could be improved remarkably if tests for dislocations were established. Dislocations are generally observed by electron microscopy, but this method is not nondestructive. We thus propose obtaining information on dislocations nondestructively before the nucleation of cracks takes place. With an indirect method, it would be possible to use the physical property which is most sensitive to dislocations.

The most sensitive properties are the magnetic ones. The relation between magnetism and dislocations has been long investigated. The most famous magnetic phenomenon due to dislocations is the roll-induced magnetic anisotropy in permalloy. 'Two different origins of induced anisotropy bave been postulated; in the first, such anisotropy is thought to be caused by magnetoelastic coupling energy due to the strain field around the dislocations, ${ }^{2,3}$ and in the second, the cause is conjectured to be the dipole-dipole interaction due to the atomic arrangement in the APB, the so-called atom pair model. ${ }^{4-7}$ These two models have progressed independently; the former model has been developed and systematized by the Stuttgart group of the Max-Planck Institute, ${ }^{3.8-11}$ and the latter has been extended to the magnetic transition due to plastic deformation by one of the present authors and his

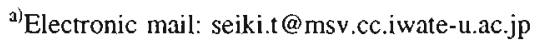

group. It has been concluded that both models are simultaneously realized in the $\mathrm{Ni}_{3} \mathrm{Fe}$-ordered alloy. ${ }^{12}$

Depending on the materials, there are three mechanisms by which the magnetic properties change due to plastic deformation. The first mechanism is found in ferromagnetic materials such as $\mathrm{Fe}, \mathrm{Ni}$, and $\mathrm{Co}$ metals. ${ }^{8-10}$ The second is applied to the magnetic transition due to plastic deformation in the intermetallic compounds and ordered alloys. ${ }^{13}$ The $\mathrm{Ni}_{3} \mathrm{Al}$ intermetallic compound is ferromagnetic, which changes to paramagnetic by plastic deformation. ${ }^{14.15}$ Another example is the $\mathrm{Fe}-\mathrm{Al}$ intermetallic compound which is paramagnetic, but becomes ferromagnetic by plastic deformations. ${ }^{16}$ This mechanism has been investigated theoretically and experimentally. ${ }^{17}$ The third mechanism is phase transformation due to plastic deformation as shown in SUS 304 stainless steel; the structure of stainless steel changes from fcc to bcc by martensitic transformation. This mechanism has been explained by Olson and Cohen and many other investigators. ${ }^{18-21}$ In the second and third types, magnetic properties such as spontaneous magnetization $M_{s}$ and Curie temperature change drastically due to plastic deformation. Only the first mechanism is considered in the present study.

The mechanism caused by the strain field due to dislocations has an influence on the magnetization curve through magnetoelastic coupling, i.e., the strain field of dislocations exerts an influence on the spin distribution through the magnetostriction. The structure-sensitive properties change due to plastic deformation in ferromagnetic materials: the initial susceptibility decreases and the coercive force $H_{c}$ and the reversible susceptibility $\chi_{r}$ increase with plastic deformation. $H_{c}$ increases by about 100 times due to plastic deformation. These phenomena were studied theoretically and experimentally considering the dislocation structure elucidated by Seeger and Kronmüller and their co-workers 3 decades ago. ${ }^{3,9.10}$ These structure-sensitive properties are highly 
TABLE I. Composition of A533B steel.

\begin{tabular}{cccccccccc}
\hline \hline A533B & $\mathrm{C}$ & $\mathrm{Si}$ & $\mathrm{Mn}$ & $\mathrm{P}$ & $\mathrm{S}$ & $\mathrm{Cu}$ & $\mathrm{Ni}$ & $\mathrm{Mo}$ & $\mathrm{Al}$ \\
\hline wt \% & 0.18 & 0.15 & 1.50 & 0.004 & 0.001 & 0.03 & 0.66 & 0.56 & 0.01 \\
\hline \hline
\end{tabular}

related to the dislocation density and its distribution. The above-mentioned researchers were mainly interested in the academic aspects of plastic deformation and concentrated on the investigation of ideal cases using single crystals. However, investigation of the relationship of these properties with dislocation density and its distribution in the case of practical materials is necessary in nondestructive tests for dislocations. One of the purposes in the present study is to apply the previous results to practical ferromagnetic materials.

One of the important and urgent areas for the application of nondestructive tests is the nuclear power station, especially the pressure vessel. The deterioration of the pressure vessel determines the lifetime of the nuclear reactor. Since most pressure vessels are made of A533B steel, in the present study the structure-sensitive properties are investigated in connection with the plastic deformation in A533B steel as well as that in standardized samples such as Fe single crystals and polycrystalline specimens.

The coercive force is a good parameter to estimate the value of $\rho ; H_{c}$ increases with the square root of $\rho .^{8.9}$ this being confirmed for single crystals with tensile tests. Fatigue tests were conducted for $\mathrm{Fe}$ single crystals by measurement of the structure-sensitive properties, in which the value of $H_{c}$ becomes saturated in the advanced stage. ${ }^{11}$ The high-field susceptibility $\chi_{r}$ is more sensitive to the dislocation density and its distribution than $H_{c}$, but the value is difficult to obtain experimentally. There are two difficulties in the application of nondestructive tests: demagnetization and the necessity of a high external field. The main purpose of the present study is to find a parameter which is more sensitive to $\rho$ than $H_{c}$ and easier to measure than the susceptibility $\chi_{r}$. This parameter, as well as $H_{c}$ and $\chi_{r}$, should be universal and be applicable to other materials such as SA508 steel, which is the other component material of the pressure vessel.

Experimental study on the relation between the structure-sensitive properties and dislocations has been conducted in single crystals. The investigation of grain boundaries is essential for the application of previous findings to the practical materials. A purpose of the present study is to investigate the grain-boundary effect on the structuresensitive properties.

\section{EXPERIMENTAL PROCEDURE}

We prepared three sheets of Fe metals and alloys: single crystals and polycrystalline samples of pure iron (99.99\%), and $\mathrm{A} 533 \mathrm{~B}$ steel. The compositions of $\mathrm{A} 533 \mathrm{~B}$ steel are shown in Table I. A533B steel is a manganese-molybdenum steel modified by increased nickel and reduced chromium content. During the manufacturing process it is austenitized between 860 and $890^{\circ} \mathrm{C}$. This is followed by water quenching and tempering processes between 650 and $665^{\circ} \mathrm{C}$ for

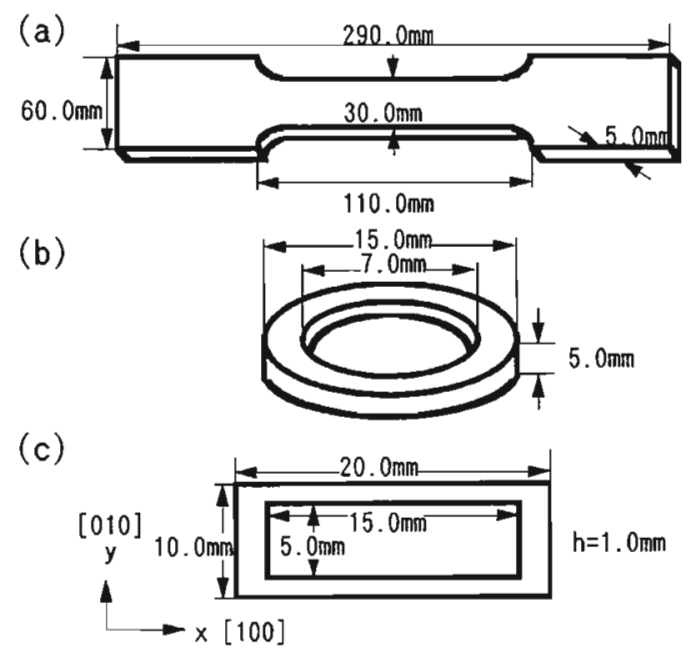

FIG. 1. Shape and size of the samples. (a) Sample size of A533B steel using the tensile test and the fatigue test. (b) Sample size of polycrystalline and A533B steel for the hysteresis loop measurement. (c) Sample size of single crystals.

139 min. The grain sizes of A533B steel and polycrystalline samples as determined by metallurgical microscopic observation are about 10 and $40 \mu \mathrm{m}$, respectively. Each sample was tensile deformed at room temperature by an Instron-type testing machine. The tensile direction of Fe single crystals is [100]. The single crystals were cut into picture frame samples with [100] and [010] frames by spark cutting for the magnetic measurement. The other samples were shaped into a doughnut form. The shapes and the sizes are shown in Fig. 1. The magnetization curves are measured using a fluxmeter at room temperature, the magnetizing and detecting coils on these samples being wound with 80 and 100 turns, respectively. It was ascertained that the measured results were independent of the number of coil turns between 50 and 150 turns. The dislocations in each sample are observed by electron microscope.

\section{STRUCTURE-SENSITIVE PROPERTIES AND THE LATTICE DEFECTS}

Structure-sensitive magnetic properties are influenced by dislocations and grain boundaries. The effect of dislocations has been studied theoretically and experimentally ${ }^{3.9}$ and the results are briefly introduced above. The effect of grain boundaries has been studied experimentally and $H_{c}$ was found to increase with the decrease of the grain size. ${ }^{22}$

\section{A. Coercive force and dislocations}

The magnetization process proceeds by domain-wall movement in a single crystal. The coercive force is given by

$$
H_{c}=\frac{1}{2 M_{s} F \cos \phi}\left(\frac{\partial E_{\omega}}{\partial x}\right)_{\max } \text {. }
$$

where $F$ is the total area of the domain wall in the unit volume, $\phi$ is the angle between the normal of the Bloch wall and $H$, and $E_{\omega}$ is the domain-wall energy depending on the position and results from the strain field of lattice defects. In a previous study, $H_{c}$ is obtained in a single magnetic do- 
main; the dislocations exert a force on the domain wall and play the role of an obstacle to the $180^{\circ}$ Bloch wall movement. This model can be applied to single crystals and polycrystalline samples with large grain sizes. $\left(\partial E_{\omega} / \partial x\right)_{\max }$ is calculated and found to be proportional to $\sqrt{\rho}{ }^{9,10} H_{c}$ depends on the distribution of dislocations. The value of $H_{c}$ in making a group consisting of $n$ dislocations becomes $\sqrt{n}$ times larger than the homogeneous distribution. ${ }^{10}$

The initial susceptibility decreases according to the inverse of the square root of $\rho$. The validity of these relations was confirmed for single crystals of $\mathrm{Ni}, \mathrm{Fe}$, and $\mathrm{Co}$ metals.

\section{B. Magnetic susceptibility and dislocations}

The magnetic moments are deflected in the vicinity of a dislocation line from the direction of easy magnetization of the magnetocrystalline anisotropy; dislocations contain the strain field in themselves and this influences the direction of magnetic moments through magnetoelastic coupling. In other words, a magnetic anisotropy exists in the vicinity of the dislocations. The magnetic anisotropy energy is a function of $\rho$, the type of dislocations, and their structure in general, i.e., $E_{\sigma}\left(\rho, \xi, \beta_{i}, \theta\right) ; \xi$ is a parameter representing the dislocation distribution, $\beta_{i}$ is the angle representing the dislocation type, and $\theta$ is the angle between $H$ and $M_{s}$.

The other property related to dislocations is magnetic susceptibility. When the deviation of the magnetic moment from the external field is small or the external field is high, the magnetization is given by

$$
M=M_{s} \cos \theta \approx M_{s}\left(1-\frac{\theta^{2}}{2}\right),
$$

and $\theta$ is given by

$$
\theta \approx \frac{1}{H M_{s}} \frac{\partial E_{\sigma}}{\partial \theta} .
$$

Then, Eq. (2) becomes

$$
M=M_{s}\left(1-\frac{c}{2 H^{2}}\right),
$$

where $c$ is given by

$$
c=\frac{1}{M_{s}^{2}}\left(\frac{\partial E_{\sigma}}{\partial \theta}\right)^{2} \text {. }
$$

The magnetic susceptibility can be written as

$$
\chi_{c}=\frac{\partial M}{\partial H}=\frac{c}{H^{3}} .
$$

The magnetic susceptibility is measured in the low-field range just above the coercive field $H_{c}$. The relation between $\chi_{c}$ and $H$ is confirmed and the parameter $c$ is obtained as a function of the applied stress. The physical meaning of $c$ will be explained in detail later. This experimental finding shows that $c$ has an intimate relation with the strain field around the dislocations. The relation of Eq. (6a) was also ascertained in the range of ferromagnetic saturation field by the Stuttgart group. ${ }^{3.9}$ We introduce the susceptibility $\chi_{r}$ in the range of saturation field to distinguish it from $\chi_{c}$. The introduction of $\chi_{r}$ helps us to understand the meaning of $c$. Equation (6a) is rewritten as

$$
\chi_{r}=\frac{\partial M}{\partial H}=\frac{c^{\prime}}{H^{3}} .
$$

The parameter $c^{\prime}$ has been calculated in simple cases. ${ }^{9}$ The magnetic moments in the vicinity of the dislocations are deflected within the exchange length. The anisotropy due to dislocations increases proportional to $\rho$. The parameter $c^{\prime}$ in Eq. (6b) is given by ${ }^{9}$

$$
c^{\prime}=\frac{9}{8} F\left(\beta_{i}\right) \frac{G^{2} b^{2} \lambda_{s}^{2} n \rho}{4 \pi M_{s}}\left(\ln \frac{R_{o}^{\prime}}{l_{H}}-2.00\right),
$$

where $G$ is the shear modulus, $\mathbf{b}$ is the Burgers vector, $\lambda_{s}$ is the magnetostriction constant, $R_{o}^{\prime}=1 /(2 \sqrt{\rho})$ is the distance within which the long-range stress field is shielded, and $l_{H}$ is the exchange length of the magnetic field. $F\left(\beta_{i}\right)$ is the anisotropy function representing the dislocation character. ${ }^{9}$ The value of $\chi_{r}$ depends on the distribution of the dislocations. The value of $\chi_{r}$ in making a group consisting of $n$ dislocations becomes $n$ times larger in comparison to the homogeneous distribution.

\section{Grain boundary and coercive force}

To date, experimental investigations between dislocations and magnetic properties have considered the ideal case of single crystals. However, the effect of grain boundaries on magnetic properties should be studied so that the above results can be applied to nondestructive tests.

The easy direction of magnetization is discontinuous in the grain boundary. The magnetic moments are deflected near the grain boundary against the exchange interaction. The grain boundary acts as an obstacle to the Bloch wall movement. Domain-wall energy $E_{\omega}$ is influenced by the different atomic structure of the grain boundary. $H_{c}$ and the initial susceptibility depend on the grain size. We can separate the effect of grain boundaries into two cases: (i) The grain size is sufficiently large enough for the Bloch wall to move in the grain. (ii) The grain size is equivalent to or smaller than the thickness of the Bloch wall and Bloch walls do not exist in the grain. The mechanism of the effect is different in the two cases. The value of $H_{c}$ becomes very large in the latter case and cannot be explained by the present experiments. We deal with the former case in the present study.

The deflection of moments exists along the grain boundary at the triple point where three grains contact and at the other multiple point where more than four grains contact each other. The Bloch wall receives the forces of $f_{a}, f_{t}$, and $f_{m}$ in the grain boundary, the triple points, and the multiple point. respectively. These forces depend on the relative crystal orientation of the grains. $H_{c}$ is given by Eq. (1). The maximum force of the domain wall by the grain boundaries is given by

$$
\left(\frac{\partial E_{\omega}}{\partial x}\right)_{\max }=\frac{k_{1}\left\langle f_{a}\right\rangle}{a^{2} d}+\frac{k_{2}\left\langle f_{t}\right\rangle}{a d^{2}}+\frac{k_{3}\left\langle f_{m}\right\rangle}{d^{3}},
$$




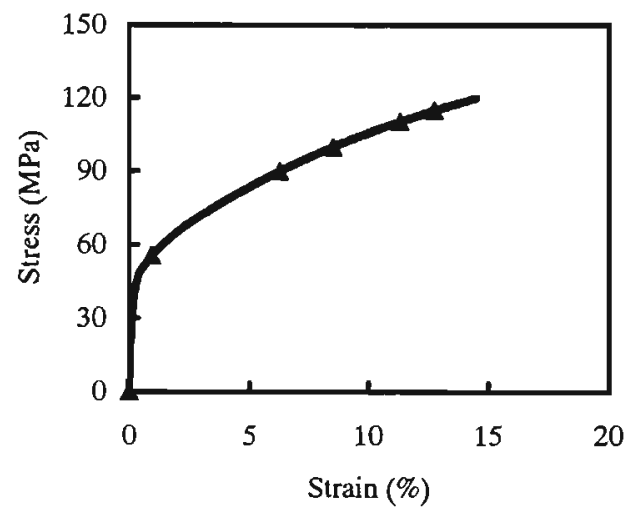

FIG. 2. Stress-strain curves of single-crystal samples. Samples were deformed by the stress indicated by the symbol $\boldsymbol{A}$.

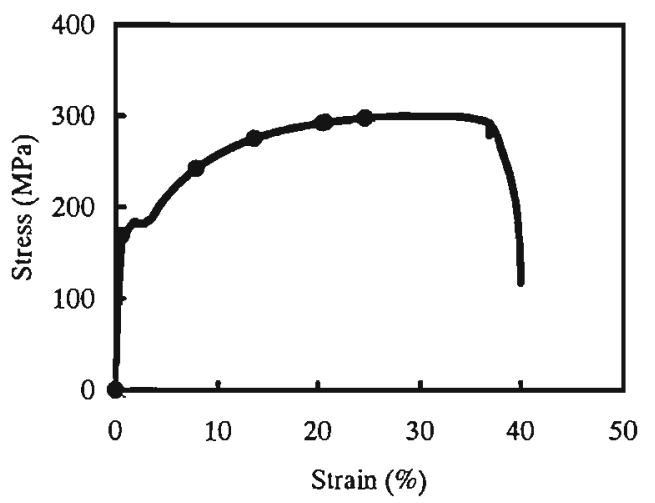

FIG. 3. Stress-strain curves of polycrystalline samples. Samples were deformed by the stress indicated by the symbol $\bullet$

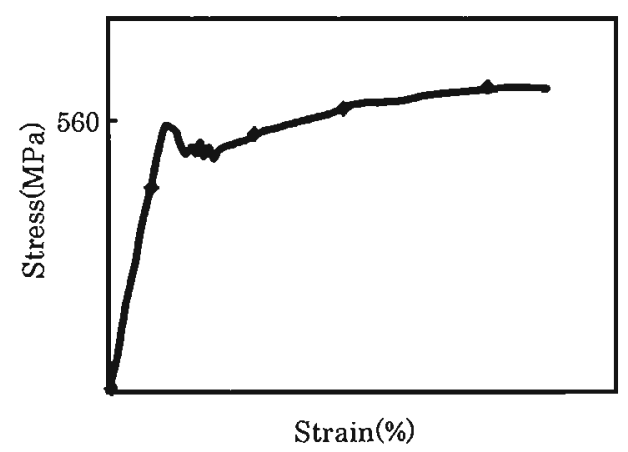

FIG. 4. Stress-strain curves of A533B steel. Samples were deformed by the stress indicated by the symbol $\bullet$

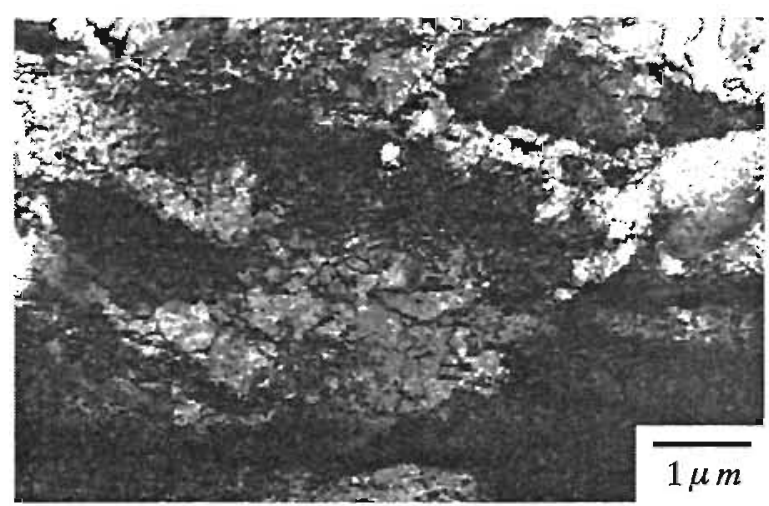

FIG. 5. Electron micrograph of the single-crystal sample with an applied stress of $110 \mathrm{MPa}$. where $d$ is the mean diameter of the grain, $k_{i}$ are constants depending on the shape of the grain boundary, and $a$ is the lattice constant. The second and third term are negligible compared with the first one and the coercive force is given by

$$
H_{c}=\frac{k_{1}\left\langle f_{a}\right\rangle}{2 M_{s} a^{2} F \cos \phi} \frac{1}{d} .
$$

$H_{c}$ is inversely proportional to $d$. This relation agrees well with the experimental result in $\alpha$-Fe metal. ${ }^{22}$

In the polycrystalline samples, dislocations and grain boundaries would be considered in the nondestructive tests. The problem of which effect is more dominant, $d$ or $\rho$, becomes important in the polycrystalline samples.

\section{EXPERIMENTAL RESULTS}

The value of $\rho$ as shown by electron microscopy is the local value and contains microscopic information on the crystal. This value, however, contains large measurement errors compared with the value of $\sigma$. The internal stress and the magnetic properties give macroscopic information on dislocations. It is reasonable to first obtain the relationship between magnetic properties and $\sigma$ and then to compare it with that shown by electron microscopy.

\section{A. Stress-strain curves}

The stress-strain curves of Fe single crystals and polycrystalline samples are shown in Figs. 2 and 3, respectively. The strain rate is $1.5 \% / \mathrm{min}$ in a single crystal and $1.2 \% / \mathrm{min}$ in polycrystalline samples. The solid triangles and circles represent the maximum stress point of each sample; five polycrystalline samples with different applied stresses were prepared. The critical shear stress in single crystals is smaller than that of polycrystalline samples. The stress-strain curves are the same as those of many previous studies.

The stress-strain curve of A533B steel is quite different from those of single and polycrystalline samples, as shown in Fig. 4. The strain rate is $1.2 \% / \mathrm{min}$. The critical shear stress is about $550 \mathrm{MPa}$, three times greater than that of the polycrystalline samples. The applied stress of $462 \mathrm{MPa}$ in A533B steel is within the elastic region. The large difference of the stress-strain curves between A533B steel and poly-

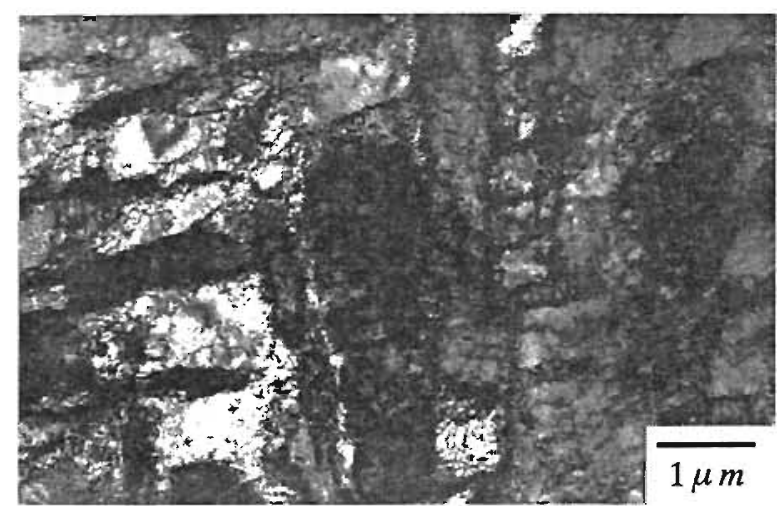

FIG. 6. Electron micrograph of the polycrystalline sample with an applied stress of $275 \mathrm{MPa}$. 


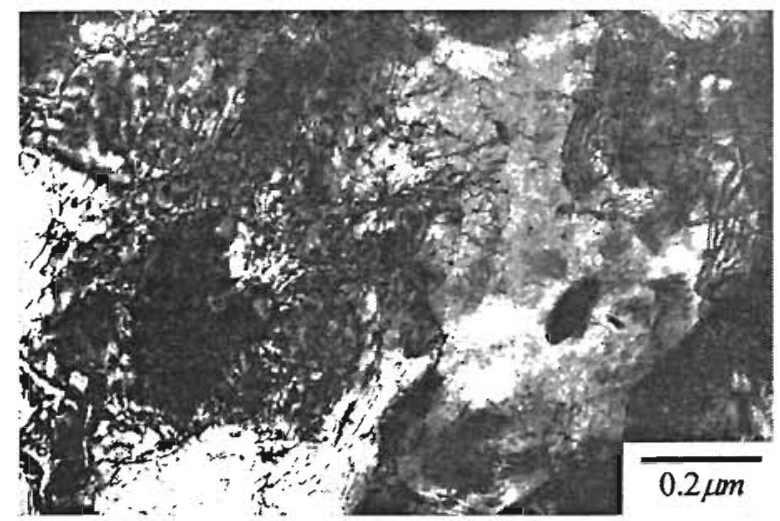

FIG. 7. Electron micrograph of an A533B steel sample before plastic deformation.

crystalline samples is caused by the dislocation density in their initial state, as shown in the next section.

\section{B. Electron microscopy observation}

Dislocations in $\mathrm{Fe}$ metals have long been investigated by many investigators. The present observation is similar to the previous ones. A few typical micrographs of dislocations are given here. Figures 5 and 6 are the electron micrographs of a single crystal sample and a polycrystalline sample with applied stress of 110 and $275 \mathrm{MPa}$, respectively. Dislocations are distributed homogeneously in both samples.

Figures 7 and 8 are electron micrographs of A533B steel before plastic deformation and with applied stress of 663 $\mathrm{MPa}$, respectively. The A533B steel contains a high value of $\rho$ initially, in which dislocations are homogeneously distributed and form a group with the same sign of the Burgers vector. In the sample with $663 \mathrm{MPa}$ stress, above which the sample was broken, dislocations form a cell structure or a band structure and their density increases remarkably.

The value of $\rho$ was measured by counting the intersection of the dislocation lines with straight lines drawn at random on photographic films. The observed value of $\rho$ may include an error of about $30 \%$ due to the uncertainty of the foil thickness and the diffraction conditions. The dependence of $\rho$ on $\sigma$ is represented in Fig. 9. A533B steel contains a high value of $\rho$ even before plastic deformation. The value of $\rho$ is $3 \times 10^{10} \mathrm{~cm}^{-2}$. The relation between $\rho$ and $\sigma$ is not smoothly connected between single crystals and the polycrystalline group, due to the grain boundary. The grain boundary contributes to the increase of $\sigma$ in plastic deformation. The differences between the polycrystalline samples and $\mathrm{A} 533 \mathrm{~B}$ steel can be attributed to the grain size.

\section{Hysteresis loops}

Figures 10 and 11 are the hysteresis loops of the single crystals plastically deformed by 0,55 , and $115 \mathrm{MPa}$, and A533B samples with plastic deformation by 0,550 , and 663 MPa, respectively. The coercive forces in these A533B steel samples are 7.92. 11.41, and $13.10 \mathrm{Oe}$, respectively. The magnetic susceptibility $\chi_{c}$ of the single crystal with 115 MPa applied stress and the A533B steel sample with 663 MPa of applied stress is plotted against $H$ above $H_{c}$ in Figs.

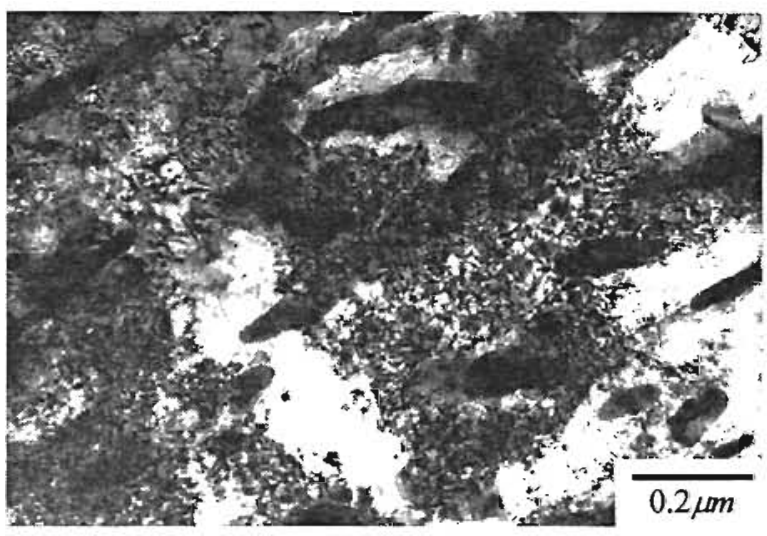

FIG. 8. Electron micrograph of an A533B steel sample with the applied stress of $663 \mathrm{MPa}$.

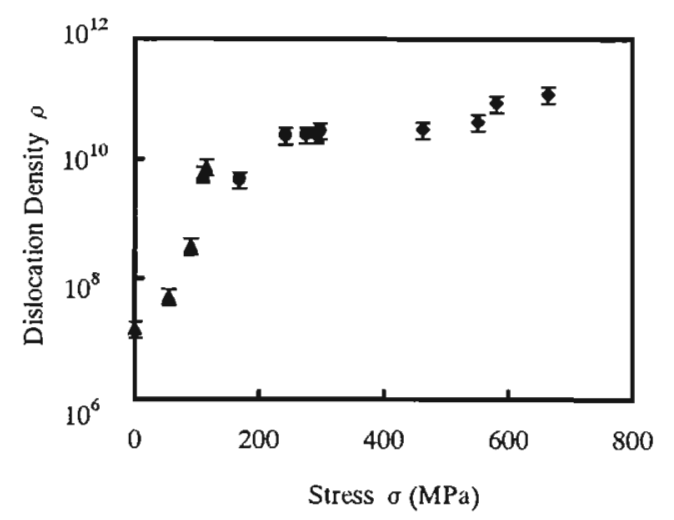

FIG. 9. Dependence of dislocation density $\rho$ on the stress $\sigma$ in single crystal $(\mathbf{A})$, polycrystalline $(\bullet)$, and A533B $(\bullet)$ steel samples.

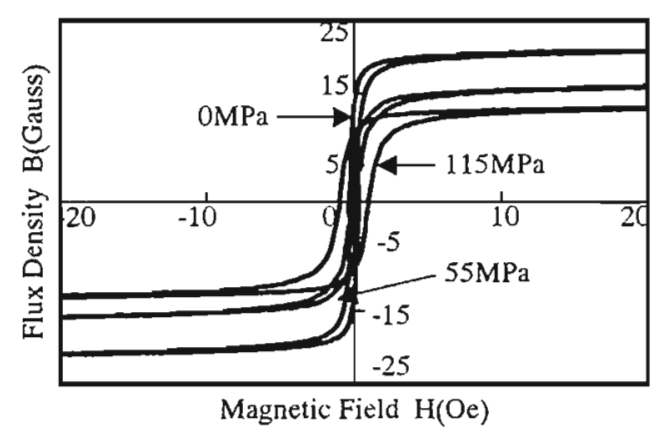

FIG. 10. Hysteresis loops of single-crystal samples with 55 and $115 \mathrm{MPa}$ of applied stress and without plastic deformation.

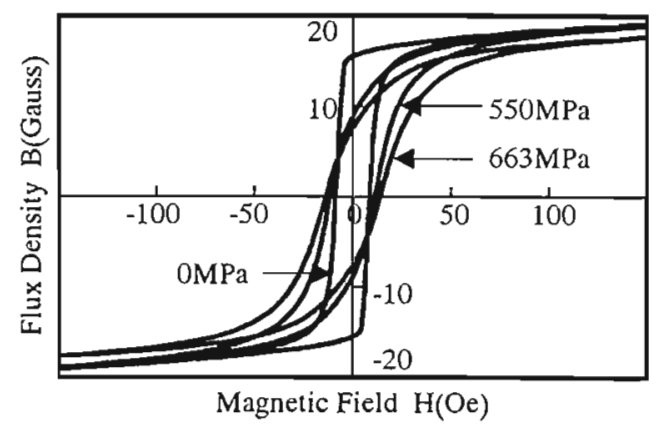

FIG. 1I. Hysteresis loops of the A533B steel samples with 550 and 663 $\mathrm{MPa}$ of applied stress and without plastic deformation. 


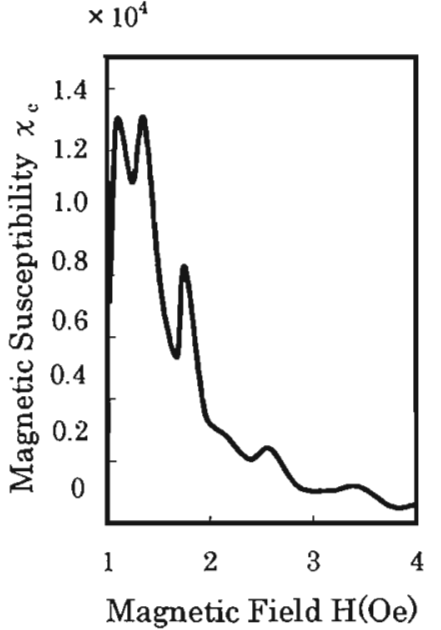

FIG. 12. Dependence of the magnetic susceptibility $\chi_{c}$ on the magnetic field above the coercive field $H_{c}$ in a single-crystal sample with an applied stress by $115 \mathrm{MPa}$.

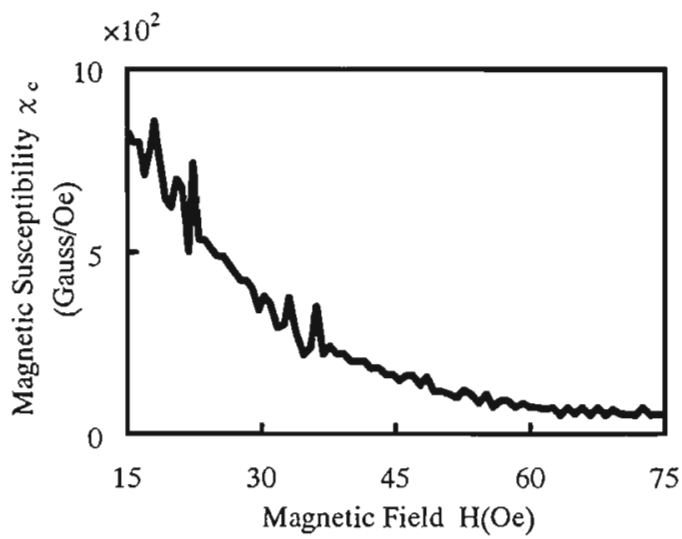

FIG. 13. Dependence of the magnetic susceptibility $\chi_{c}$ on the magnetic field above the coercive field $H_{c}$ in the A533B steel sample with an applied stress by $663 \mathrm{MPa}$.

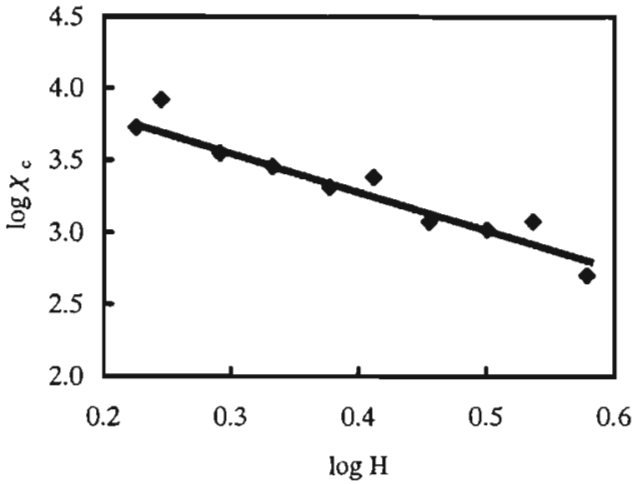

FIG. 14. Dependence of the magnetic susceptibility $\chi_{c}$ on the magnetic field above the coercive field $H_{c}$ in a single-crystal sample with plastic deformation by $115 \mathrm{MPa}$. Both are plotted by the logarithmic values. The slope of the straight line -3 indicates the validity of Eq. (6a).
12 and 13, respectively. The same property of the single crystal with $115 \mathrm{MPa}$ applied stress is plotted in logarithm values to test the relation of Eq. (6a) in Fig. 14 and that of A533B steel with $663 \mathrm{MPa}$ in Fig. 15. The relation of Eq. (6a) is valid in the limited region, $p_{1} H_{c}<H<p_{2} H_{c}$, where $p_{1}$ and $p_{2}$ are parameters representing the limited magnetic field. The values of $p_{1}, p_{2}$, and $H_{c}$ are shown in Table $\Pi$. In the field smaller than $p_{1} H_{c}$, the relation of Eq. (3) is not valid because the approximation, $\sin \theta=\theta$, is not obeyed. Above $p_{2} H_{c}, X_{c}$ decreases rapidly with the increase of $H$ and the relation cannot be verified in the present measurement. The relation of Eq. (6a) is found in all the samples. The parameter $c$ is obtained for each sample and is shown in Table II. The smallest value of $c$ is $5 \times 10^{3} \mathrm{GOe}^{2}$ in the undeformed sample of single crystals and its value is 1.5 $\times 10^{7} \mathrm{G} \mathrm{Oe}^{2}$ in the A533B sample with an applied stress of $680 \mathrm{MPa}$.

The coercive force increases with the increase of $\sigma$ as shown in Fig. 16. $H_{c}$ values are arranged in one curve without a clear discontinuity between the different kinds of samples. A discontinuity, however, is observed in the slope of the curve between the single crystal and the polycrystalline samples, but no discontinuity in the slope is observed between the polycrystalline and A533B steel samples.

The dependence of $c$ on $\sigma$ is represented in Fig. 17. The parameter $c$ is plotted on a logarithmic scale. The relation between $c$ and $\sigma$ can be expressed by one straight line with small scattering, which means that $c$ depends on only $\sigma$ and is independent of the kind of sample. Internal stress is caused by the grain boundary as well as by the dislocations. Both the dislocations and the grain boundary determine the value of $c$.

\section{DISCUSSION}

Shear stress $\sigma$ has a simple relation with the dislocation density and its distribution in a single crystal: i.e., $\rho \propto \sigma$ $-\sigma_{o}$ in stage I where $\sigma_{o}$ is the critical shear stress, and $\rho$ $\propto \sigma^{2}$ in stage II. In the polycrystalline samples, however, the yield shear stress $\tau$ depends on the grain size as well as on the dislocation density, i.e., the Hall-Petch relation, $\tau=\tau_{o}$ $+k_{y} / \sqrt{d}$, where $\tau_{o}$ is the yield shear stress of a single crystal

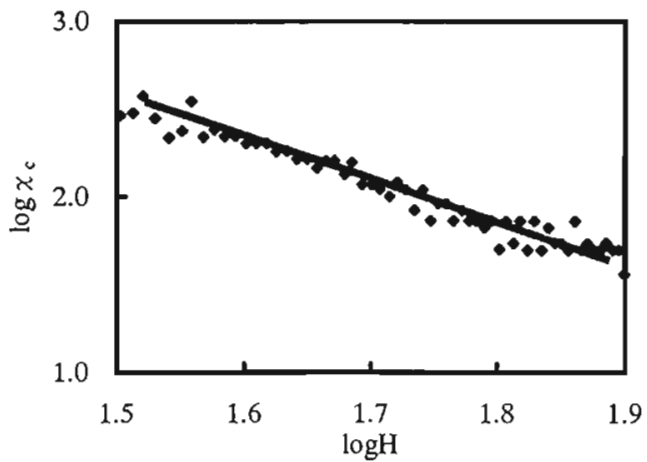

FIG. 15. Dependence of the magnetic susceptibility $\chi_{c}$ on the magnetic field above the coercive field $H_{c}$ in an A533B steel sample with plastic deformation by $663 \mathrm{MPa}$. Both are plotted by the logarithmic values. The slope of the straight line -3 indicates the validity of $\mathrm{Eq}$. (6a). 
TABLE II. Coercive force $H_{c}$ and the parameter $c$ in Fe single crystals, Fe polycrystalline samples, and A533B steel. The magnetic susceptibility $\chi_{c}$ is measured in the applied field $H$, between $p_{1} H_{c}$ and $p_{2} H_{c}$. The parameter $c$ has a relation with $H ; \chi_{c}=c / H^{3}$. Fatigue tests were also performed in A533B steel.

\begin{tabular}{lccccc}
\hline \hline & $\sigma(\mathrm{MPa})$ & $H_{c}(\mathrm{Oe})$ & $p_{1}$ & $p_{2}$ & $c\left(\mathrm{G} \mathrm{Oe}^{2}\right)$ \\
\hline & 0 & 0.22 & 4.98 & 5.57 & $5.01 \times 10^{3}$ \\
Single & 55 & 0.33 & 4.51 & 5.35 & $1.00 \times 10^{4}$ \\
crystals & 90 & 0.46 & 3.47 & 4.80 & $1.58 \times 10^{4}$ \\
& 100 & 0.75 & 2.92 & 4.10 & $3.16 \times 10^{4}$ \\
& 110 & 0.96 & 2.16 & 3.91 & $3.98 \times 10^{4}$ \\
& 115 & 0.97 & 2.21 & 3.89 & $4.47 \times 10^{4}$ \\
Poly- & 0 & 0.68 & 3.94 & 5.54 & $1.26 \times 10^{4}$ \\
crystalline & 168 & 1.06 & 2.78 & 4.63 & $3.98 \times 10^{4}$ \\
samples & 242 & 2.58 & 1.50 & 2.35 & $1.58 \times 10^{5}$ \\
& 272 & 2.99 & 1.54 & 2.28 & $1.58 \times 10^{5}$ \\
& 292 & 3.30 & 1.62 & 2.31 & $2.00 \times 10^{5}$ \\
& 293 & 3.38 & 1.78 & 2.40 & $2.51 \times 10^{5}$ \\
& 297 & 3.67 & 1.74 & 2.34 & $2.82 \times 10^{5}$ \\
& & & & & \\
A533B steel & 0 & 7.92 & 1.60 & 4.04 & $1.60 \times 10^{6}$ \\
& 462 & 11.41 & 2.80 & 4.38 & $7.10 \times 10^{6}$ \\
& 550 & 13.10 & 3.05 & 4.81 & $1.40 \times 10^{7}$ \\
& 580 & 13.10 & 3.05 & 4.81 & $1.50 \times 10^{7}$ \\
& 663 & 7.57 & 1.48 & 3.73 & $1.78 \times 10^{6}$ \\
& $500 \mathrm{MPa}, 50$ cycles & 9.78 & 1.29 & 6.45 & $7.94 \times 10^{6}$ \\
\hline \hline
\end{tabular}

and $k_{y}$ is a constant. The shear stress in this case yields information on the lattice structure, including the lattice defects.

The magnetic properties change with the lattice defects, and the relation between $H_{c}$ and $\sigma$ is investigated in the three kinds of samples. Figure 16 reveals that a discontinuity appears in the slope of the curve between the single crystals and polycrystalline samples. The value of $H_{c}$ in the polycrystalline samples, even before plastic deformation, is much larger than that of the single crystals. The initial value of $\rho$ in the polycrystalline samples is so small that the dislocations do not contribute to the value of $H_{c}$, which is reflected mainly by the grain boundary. The grain size of the polycrystalline samples is about $50 \mu \mathrm{m}$, which is much smaller than the critical size of $500 \mu \mathrm{m} .^{22}$ The mechanism of coercive force in the polycrystalline samples is explained by another model, namely, the rotation of magnetic moments within the grain dominates the magnetization process. The value of $H_{c}$ in the single-domain model is much larger than the present

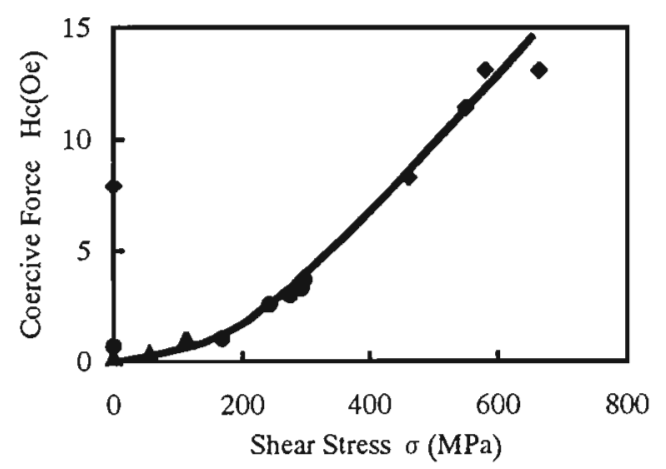

FIG. 16. Dependence of the coercive force $H_{c}$ on the applied stress $\sigma$ in single crystal (A), polycrystalline $(\bullet)$, and A533B $(\bullet)$ steel samples. results. The mechanism below the critical grain size is under consideration. The following conclusions can be drawn from the experimental results. The relation can be represented by one curve though more than two mechanisms exist depending on the grain size. The coercive force is available as a parameter to estimate the process of deterioration due to dislocations.

The physical origin of $\chi_{r}$ is clear according to this model; the deffection of magnetic moments around the dislocations within the exchange length is important in $\chi_{r}$. The exchange lengths of stray field, the magnetocrystalline energy, and the magnetic field are given, respectively, by ${ }^{9}$

$$
l_{S}=\sqrt{\frac{2 A}{2 \pi M_{s}^{2}}}
$$

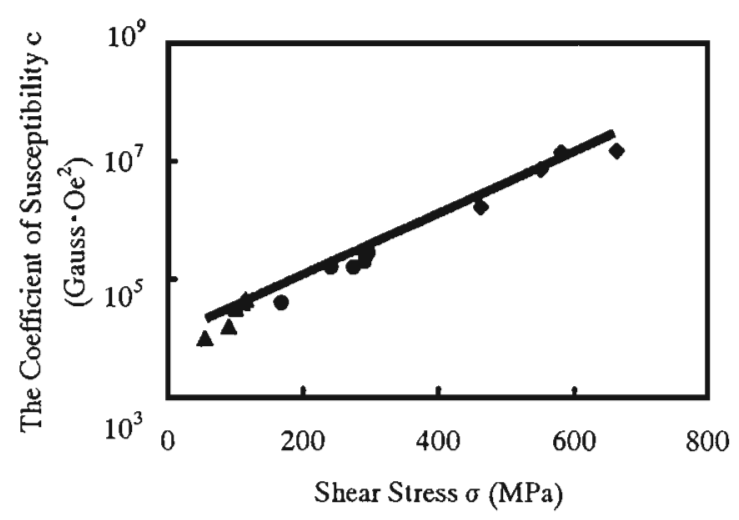

FIG. 17. Dependence of the coefficient of susceptibility $c$ on the applied stress $\sigma$ in single crystal $(\boldsymbol{\Delta})$, polycrystalline $(\bullet)$, and A533B $(\bullet)$ steel samples. $c$ is plotted in the logarithmic scale. 


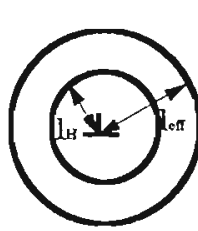

(a)

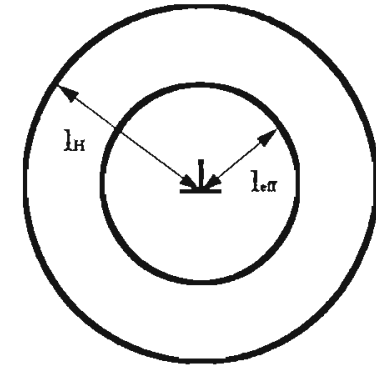

(b)
FIG. 18. Comparison of $\chi_{c}$ and $\chi_{r}$. (a) In the range of approach to the saturation field, the exchange length of the field $l_{H}$ is comparable to the effective exchange length $l_{\mathrm{cff}}$ and the susceptibility $\chi_{r}$ of magnetic moments within $l_{H}$ is measured. (b) When the applied field is small, $l_{H}$ is much larger than $l_{\mathrm{eff}}$ and the moments near $l_{H}$ contribute to the susceptibility $\chi_{c}$

$$
l_{K}=\sqrt{\frac{A}{K_{1}}},
$$

and

$$
l_{H}=\sqrt{\frac{2 A}{H M_{s}}},
$$

where $A$ is the exchange constant and $K_{1}$ is the first magnetocrystalline anisotropy constant. The effective exchange length $l_{\mathrm{eff}}$ is defined by ${ }^{9}$

$$
\frac{1}{l_{\text {eff }}}=\frac{1}{l_{S}}+\frac{1}{l_{K}}+\frac{1}{l_{H}} \text {. }
$$

The values of $l_{S}, l_{K}$, and $l_{H}$ for $\alpha$-Fe at 1000 Oe are 3.3, 20.9 , and $15.3 \mathrm{~nm}$, respectively. The exchange length of the field plays a role only within the law of approach to saturation at room temperature. For small fields, the magnetic moments around the dislocations are determined by $l_{s}$ and $l_{K}$. The value of $\chi_{r}$ can be experimentally obtained in the range of approach to magnetization above $1000 \mathrm{Oe}$.

There is a difference in the applied field between $\chi_{c}$ and $\chi_{r}$. The value of $\chi_{c}$ is obtained in the weak field between 2 and $50 \mathrm{Oe}$. The mechanism of $\chi_{c}$ is different from that of $\chi_{r}$, but both are reflective of the deflection of magnetic moments around the dislocations. The strain field of a dislocation decreases inversely with the distance $r$ from the dislocation core. The magnetic moments within the strain field of a dislocation, being far from the core, turns to the external field though the field is weak. The moments which are located at $r=l_{H} \quad\left(l_{\mathrm{eff}} \ll l_{H}\right)$ contribute to the susceptibility $\chi_{c}$, as shown in Fig. 18(b). Though the susceptibility due to the moments at far distances is weak, the observed value is large. since the number of turned moments increases proportional to $r^{2}$. The number of moments which contribute to $\chi_{c}$ is in proportion to $2 \pi \rho r d r$ when the dislocations separate sufficiently.

The magnetization at the field where $\chi_{c}$ is obtained decreases with the increases of $\sigma$ as shown in Figs. 10 and 11 . The magnetic moments within $l_{\text {eff }}$ do not turn to the applied field because the moments are firmly fixed by the magnetoelastic coupling energy. The applied field is too low to affect the deflection of moments within $l_{\text {eff }}$. These firmly fixed moments do not contribute to the value of magnetization in low fields. The number of fixed moments is proportional to $\rho$. Higher applied fields are required to rotate the firmly fixed moments within $l_{\text {eff }}$ parallel to the external field. The magnetic susceptibility $\chi_{r}$ can be measured in the high field, where $l_{\text {eff }} \sim l_{H}$ [see Fig. 18(a)]. The susceptibility $\chi_{c}$ has a different behavior; the moments outside and far from the region of $l_{\text {eff }}$ give the value of $\chi_{c}$. The detailed relation between $\chi_{r}$ and $\chi_{c}$ should become clear in the following.

The grain boundary may exert two influences on $\chi_{c}$. The value of $\chi_{c}$ depends on the grain size, since the magnetic moments are deflected within the grain boundary. The other effect is an indirect influence: dislocations pile up at the grain boundary. The former effect is independent of plastic deformation but the latter depends on deformation. The latter effect is related to the distribution of dislocations. These effects of dislocations and the grain size also affect the internal stresses. So the relation between $c$ and $\sigma$ is significant in comparison with that of $c$ and $\rho$. The separation of the two effects, due to dislocations and grain boundaries, would be interesting from the academic viewpoint, but is not necessary for the nondestructive tests, since both are the cause of cracks.

The mechanism of $\chi_{c}$ is due to the rotation of the magnetic moments and the value of $c$ is independent of the kinds of samples; the magnetostriction constant is the same and the modulus of rigidity is nearly the same in the present samples. The relation between $c$ and $\sigma$ is sufficiently simple to be represented by a single line as shown in Fig. 17. This means that the parameter $c$ depends only on the internal structures, such as crystal structures and lattice defects. It is independent of the external conditions such as the methods of plastic deformation, tensile deformation, compression, rolling. or fatigue tests. This is easily understood from the fact that the dislocations in A533B steel are first induced by rolling. The independence of $c$ from the external condition is an important factor in the use of nondestructive tests.

For the purpose of confirmation, an A533B steel sample was fatigue tested under conditions of $500 \mathrm{MPa}$ in 50 cycles and of $510 \mathrm{MPa}$ in 500 cycles, and the values of $c$ and $\rho$ were obtained by the same method as that used with the previous samples. The obtained values are consistent as to the relations $\rho \sim \sigma$ in Fig. 9 and $c \sim \sigma$ in Fig. 17; the value of $c$ is $7.94 \times 10^{6} \mathrm{G} \mathrm{Oe}^{2}$. which is between those of 550 and 580 $\mathrm{MPa}$, and the value of $H_{c}$ is $9.78 \mathrm{Oe}$, which is between those of 462 and $550 \mathrm{MPa}$. The value of $\rho$ is $7 \times 10^{10} \mathrm{~cm}^{-2}$ between the samples of 550 and $580 \mathrm{MPa}$. The relation between $\rho$ and $c$ is more consistent than that between $\rho$ and $H_{c}$. One of the reasons for this is the fact that the value of $c$ is more accurate than $H_{c}$. The inconsistency of $H_{c}$ and $c$ values is attributed to the dislocation distribution. The dislocations form the cell structure in the sample with fatigue tests of $510 \mathrm{MPa}$ in 500 cycles, which is different from that of the tensile samples. The value of $c$ would indicate a larger value in the distribution making a group than in the homogenous distribution. In the samples with $500 \mathrm{MPa}$ and 50 cycles, no difference is detected in the electron microscopy observation compared with the undeformed sample. The 
value of $H_{c}$ is smaller than that of the sample without deformation. The small value of $H_{c}$ depends on the initial condition of the samples, since the samples of the fatigue tests were purchased from a different source. The value of $c$ in the samples with $500 \mathrm{MPa}$ and 50 cycles is larger than that without deformation. Dislocations tend to form a cell structure to a slight extent due to the fatigue tests. The small change of the dislocation distribution increases the value of $c$. The parameter $c$ is very sensitive to dislocations, especially to their distribution.

The parameter $c$ is more sensitive to lattice defects than $H_{c}$. The smallest value of $c$ is $5 \times 10^{3} \mathrm{GOe}^{2}$ in a single crystal without plastic deformation and the largest value is $1.5 \times 10^{7} \mathrm{G} \mathrm{Oe}^{2}$ in A533B steel with shear stress of $663 \mathrm{MPa}$. The ratio of the largest value to the smallest is $3 \times 10^{3}$ in the parameter $c$. The extreme ratio in $H_{c}$ is 60 . The high sensitivity of $c$ is good for application to nondestructive tests. The susceptibility $\chi_{r}$ is also sensitive to lattice defects such as the value of $\rho$. The sensitivity of $\chi_{r}$ is nearly the same as that of $\chi_{c}$. The susceptibility $\chi_{c}$ has an advantage the application to nondestructive tests compared with $\chi_{r}$. The applied field for the measurement is much lower in $\chi_{c}$ than in $\chi_{r}$; the former is $50 \mathrm{Oe}$ at most and the latter is more than $1000 \mathrm{Oe}$. Both, however, have a disadvantage in that the demagnetization factor should be considered, though it is unnecessary for the coercive force. This demagnetization problem can possibly be overcome in the process of equipment development and design.

The value of $\rho$ increases about 10 times due to the applied stress of $663 \mathrm{MPa}$ in A533B steel, but the value of $H_{c}$ increases only $60 \%$, though an increase by a factor of 3 times is expected theoretically. The value of $H_{c}$ reflects the largest value of the local strain field of dislocations. Accordingly, within the regions of high dislocation density, it increases only modestly, whereas in the low-density region, the density increases significantly. The same tendency was observed in a previous study, ${ }^{11}$ where the value of $H_{c}$ became saturated for high dislocation density. The value of $c$ reflects the increase of $\rho$ directly and increases as much as that of $\rho$. The parameter $c$ would be effective in the investigation of high dislocation density such as the nucleation of cracks.

\section{ACKNOWLEDGMENTS}

The authors are indebted to Professor X. G. Li, Dr. H. Hatafuku, and K. Konishi for their help with the magnetic measurements. The authors would like to express their thanks to Professor Uesaka of Tokyo University for the offer of A533B steel and to Dr. Ara in the Japan Atomic Energy Research Institute for his valuable discussion. This work was supported by a Grant-in-Aid for Scientific Research (B) and by financial and technical aid from the Japan Atomic Energy Research Institute.

${ }^{1}$ W. Six. J. L. Snoek, and W. G. Burgers, Ingenieurs 49, E195 (1934).

${ }^{2}$ R. Becker and W, Döring, Ferromagnetismus (Springer, Berlin, 1938).

${ }^{3}$ A. Seeger, H. Kronmüller, H. Rieger, and H. Träuble, J. Appl. Phys. 35. 740 (1964).

${ }^{4}$ S. Chikazumi, K. Suzuki, and H. Iwata. J. Phys. Soc. Jpn. 12, 1295 (1957).

${ }^{5}$ S. Takahashi. Phys. Status Solidi A 42. 201 (1977).

${ }^{6}$ S. Takahashi, Phys. Status Solidi A 42. 529 (1977).

${ }^{7}$ S. Takahashi. Phys. Status Solidi A 45. 133 (1978).

${ }^{8}$ H. Kronmüller. Can. J. Phys. 45, 631 (1967).

${ }^{9}$ H. Kronmüller, Int. J. Nondestruct. Testing 3, 315 (1972).

${ }^{10} \mathrm{H}$. Träuble. in Magnetism and Metallurgy, edited by A. E. Berkowitz and E. Kneller (Academic, New York, 1969). Chap. XIII, p. 621.

${ }^{11} \mathrm{H}$. Mughrabi, R. Kütterer, K. Lubitz, and H. Kronmüller. Phys. Status Solidi A 38. 261 (1976).

${ }^{12} \mathrm{~S}$. Takahashi and H. Kronmüller, Phys. Status Solidi A 61, 207 (1980)

${ }^{13} \mathrm{~S}$. Takahashi and K. Ikeda, Phys. Rev. B 28, 5225 (1983).

${ }^{14}$ F. R. De Boer, C. J. Schinkel, J. Biersterbos, and S. Proost, J. Appl. Phys. 40. 1049 (1969).

${ }^{15}$ S. Takahashi, A. Chiba, and A. Y. Takahashi, J. Phys.: Condens. Matter 6. 10795 (1994).

${ }^{16}$ G. P. Huffman and R M. Fisher, J. Appl. Phys. 38, 735 (1967)

${ }^{17}$ S. Takahashi and Y. Umakoshi. J. Phys.: Condens. Matter 3. 5805 (1991).

${ }^{18}$ R. P. Reed and C. J. Guntner. Trans. Metall. Soc. AIME 230, 1713 (1964).

${ }^{19} \mathrm{G}$. B. Olson and M. Cohen, J. Less-Common Met. 28, 107 (1972).

${ }^{20}$ S. S. Hecker, M. G. Stout. K. P. Staudhammer, and J. L. Smith, Metall. Trans. A 13A. 619 (1982).

${ }^{21}$ G. B. Olson and M. Cohen. Metall. Trans. A 6A. 791 (1975)

${ }^{22}$ J. Degauque, B. Astie, J. L. Porteseil, and R. Vergne. J. Magn. Magn. Mater. 26. 261 (1982). 